\title{
3D Multi-Material Printing of an Anthropomorphic, Personalized Replacement Hand for Use in Neuroprosthetics Using 3D Scanning and Computer-Aided Design: First Proof-of-Technical-Concept Study
}

\author{
Jana Baron, Simon Hazubski and Andreas Otte *(D) \\ Peter Osypka Institute of Medical Engineering, Department of Electrical Engineering, \\ Medical Engineering and Computer Science, Offenburg University, Badstr. 24, D-77652 Offenburg, Germany; \\ jbaron@stud.hs-offenburg.de (J.B.); simon.hazubski@hs-offenburg.de (S.H.) \\ * Correspondence: andreas.otte@hs-offenburg.de; Tel.: +49-781-205-338
}

Received: 29 October 2020; Accepted: 17 December 2020; Published: 18 December 2020

\begin{abstract}
Background: This paper presents a novel approach for a hand prosthesis consisting of a flexible, anthropomorphic, 3D-printed replacement hand combined with a commercially available motorized orthosis that allows gripping. Methods: A 3D light scanner was used to produce a personalized replacement hand. The wrist of the replacement hand was printed of rigid material; the rest of the hand was printed of flexible material. A standard arm liner was used to enable the user's arm stump to be connected to the replacement hand. With computer-aided design, two different concepts were developed for the scanned hand model: In the first concept, the replacement hand was attached to the arm liner with a screw. The second concept involved attaching with a commercially available fastening system; furthermore, a skeleton was designed that was located within the flexible part of the replacement hand. Results: 3D-multi-material printing of the two different hands was unproblematic and inexpensive. The printed hands had approximately the weight of the real hand. When testing the replacement hands with the orthosis it was possible to prove a convincing everyday functionality. For example, it was possible to grip and lift a 1-L water bottle. In addition, a pen could be held, making writing possible. Conclusions: This first proof-of-concept study encourages further testing with users.
\end{abstract}

Keywords: amputee; anthropomorphic hand replacement; 3D multi-material printing; 3D light scanning; computer-aided design; neuroprosthetics; personalization

\section{Introduction}

Nowadays there are many different ways to design and manufacture a prosthesis for the upper limbs. The approaches to control a prosthesis can be roughly divided into two categories: non-invasive and invasive. A fascinating example for an invasive approach is the linking of intracortical recorded signals to the activation of the forearm muscles so that intact muscles can be controlled again [1]. On the other hand, there are some non-invasive concepts that use hybrid systems based on electroencephalography (EEG) and electrooculography (EOG) or electromyography (EMG) to control the prostheses [2,3]. Such concepts are impressive, but they are technically complex and associated with high costs and a high learning effort for the patient $[4,5]$, so they are not suitable for every patient. Depending on the patient's life circumstances, there is a great desire for a functional, but as simple and inexpensive as possible prosthesis [4], which serves as a support in everyday life. For this 
reason, open communication between engineers and physicians is essential for the development of neuroprosthetics for the upper limb [6].

Some non-invasive concepts have already shown that even simple approaches can produce convincing results. These include, for example, body-powered prosthesis, which are cost-effective and allow the user to grip an object by moving a body part such as the shoulder [7]. In addition, 3D-printed prostheses have also been convincing with their cost-effective production for several years now. These include the Phoenix Hand from the e-NABLE organization, which is based on design data and controlled by wrist movement [8]. In other projects, 3D printing has proven to be a remarkable alternative to traditional methods such as casting. For example, a controller-controlled, sensorimotor finger system based on the reconstruction of the first "Iron Hand" of Götz von Berlichingen was produced by using a multi-material 3D printer [9].

This paper presents another approach to a hand prosthesis in which multi-material 3D printing plays a role. The hand prosthesis consists of a combination of a 3D-printed replacement hand and a commercially available electric orthosis, which is used e.g., by quadriplegics and allows gripping. Unlike the 3D printed prosthetics mentioned above, the 3D printed replacement hand is based on 3D scanning technology. If available, the healthy hand of a hand amputee is scanned and mirrored with a 3D structured light scanner, resulting in a 3D model of an anthropomorphic replacement hand that looks like the patient's own hand. To enable the replacement hand to move through the orthosis, a flexible material must be selected for the part of the hand above the wrist. The wrist, on the other hand, should be made of strong material to give the hand stability.

In the following, two concepts are presented that show how the combination of a replacement hand and orthosis works.

\section{Results}

\subsection{Selection of the Material for the Replacement Hand}

To select the material of the flexible part of the replacement hand, five individual fingers were printed with different materials. The main material is the flexible, rubber-like photopolymer Agilus30. By mixing Agilus30 with the rigid material VeroWhite, the properties of the material change. These properties were determined by bending the fingers manually (J.B.) and are shown in Table 1.

Table 1. Properties of the materials at increasingly high mixing ratios of Agilus30 with VeroWhite.

\begin{tabular}{ccccc}
\hline Finger & Shore-A-Value & Flexibility and Effort & Tearing & Strength \\
\hline 1 & 30 & Full flexion with minimal force & no & soft, unnatural \\
2 & 40 & Full flexion with average force & no & soft, unnatural \\
3 & 50 & Full flexion with average force & no & medium, natural \\
4 & 60 & Full flexion with above average force & no & rigid, natural \\
5 & 70 & Full flexion with maximum force & yes & rigid, unnatural \\
\hline
\end{tabular}

The material chosen for the fully flexible part of the replacement hand was Finger 1, printed with pure Agilus30, although Finger 3 and Finger 4 feel most natural in terms of strength. When testing the fingers with the orthosis, Finger 1 could be flexed the most as the least force was required. In addition, no cracks were formed. The stronger flexion increases the number of objects that can be gripped with the prosthesis, which is why the criterion of functionality rather than strength prevailed in this case. The wrist was printed with the photopolymer VeroWhite. In total the replacement hand weighs $415 \mathrm{~g}$. The results of the complete replacement hand are shown in Figure 1. 


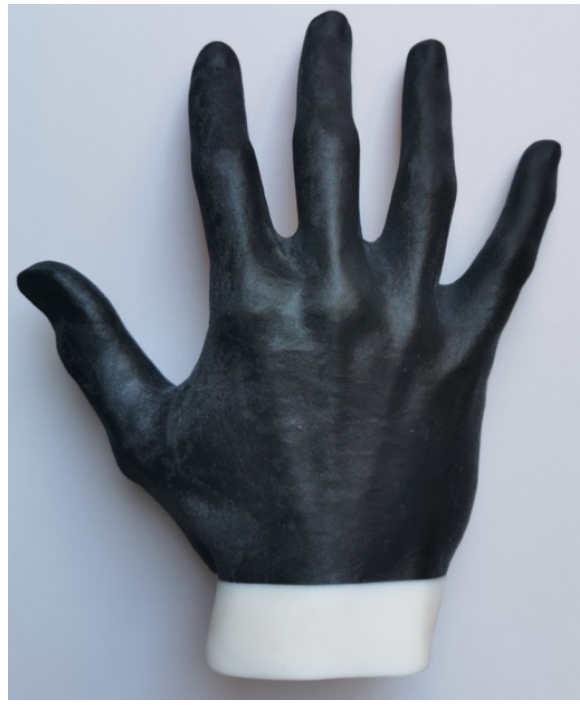

(a)

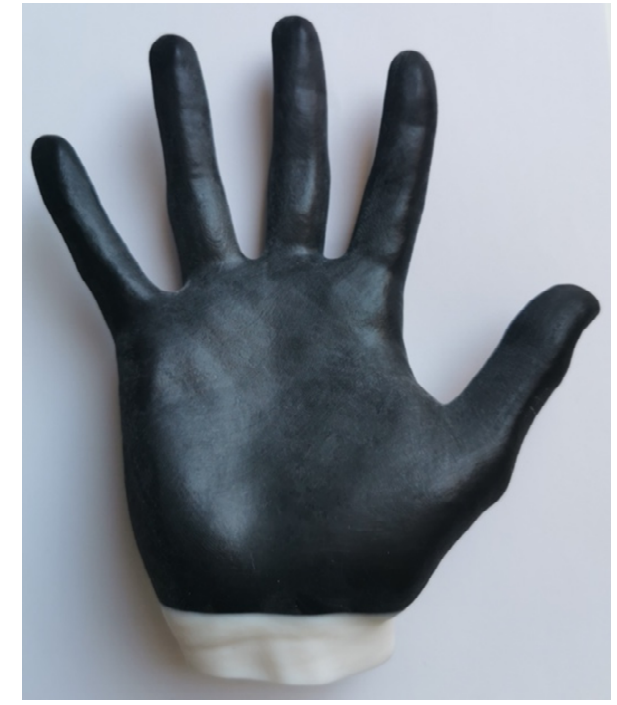

(b)

Figure 1. 3D printing of the replacement hand. (a) Replacement hand from dorsal view; (b) replacement hand from palmar view.

In addition to the hand, which is made entirely of the flexible material above the wrist, another replacement hand was printed. This contains a simplified skeleton made of rigid material within the flexible part. Figure 2 shows the replacement hand with the constructed skeleton consisting of simplified phalanges and a block. The phalanges and the block are connected. The strong structure gives the replacement hand more stability as well as a more natural feeling and flexion of the fingers. In contrast to the replacement hand without a skeleton, the fingers can only be bent in places where the phalanges are not present. The replacement hand has a total weight of $419 \mathrm{~g}$.

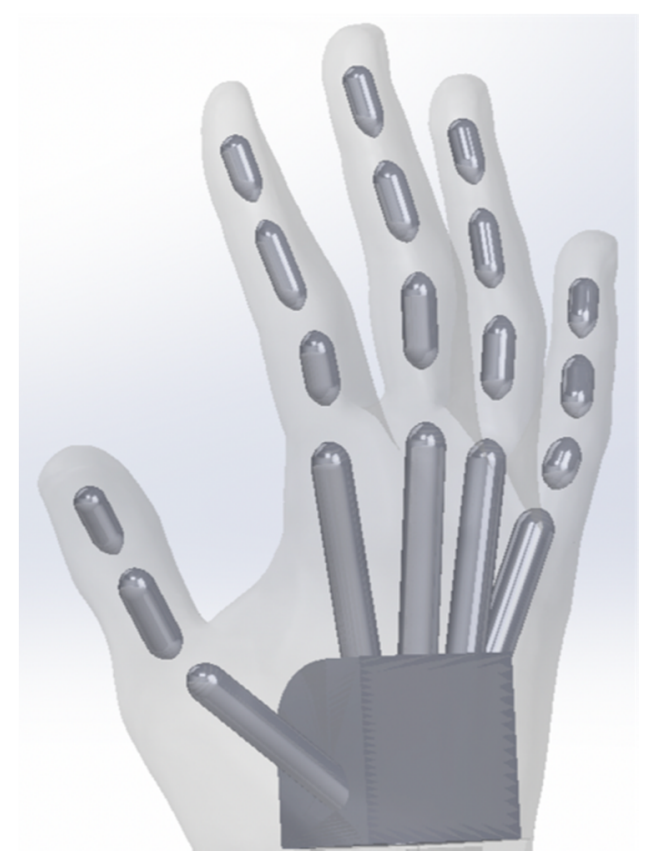

Figure 2. CAD model of the skeleton from simplified phalanges, which is placed inside the replacement hand. 


\subsection{Functionality Tests of the Replacement Hand}

Both replacement hands were tested in combination with the orthosis to determine whether the prosthesis could be used in various everyday situations. Figure 3 shows the setup of the prosthesis. The orthosis can actuate the index and middle finger. The motor of the orthosis enables the simultaneous opening and closing of the index finger and middle finger. The thumb remains rigid. A Bluetooth remote control is used to control the orthosis by pressing two buttons.

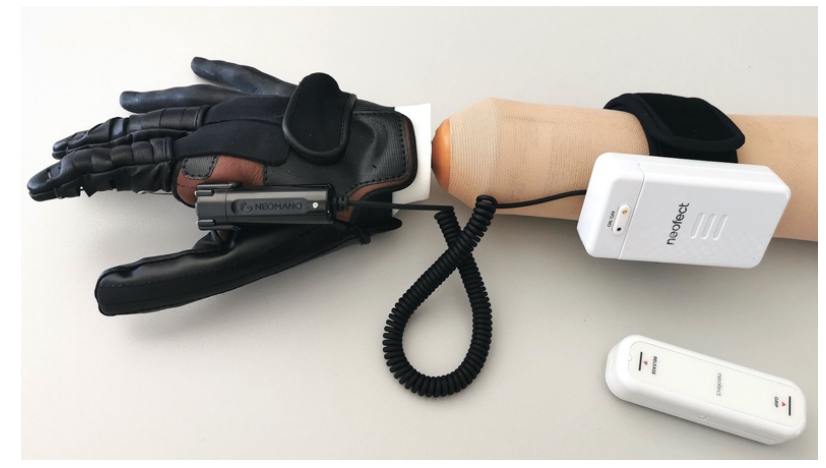

Figure 3. Assembly of the first prosthesis: Combination of the replacement hand and the orthosis. The control unit of the orthosis is attached to the Armliner.

It is possible to grip and hold various objects with the prosthesis. The flexible material withstands several bending processes. One of the objects is a full 1-L bottle, which is gripped and held up. This is shown in Figure 4 and in Supplementary Materials Video S1. Other everyday situations such as opening a door or writing with a pen are also shown in Video S1. The tests have demonstrated that the hand prosthesis can be used to perform various grips that are useful for managing everyday life.

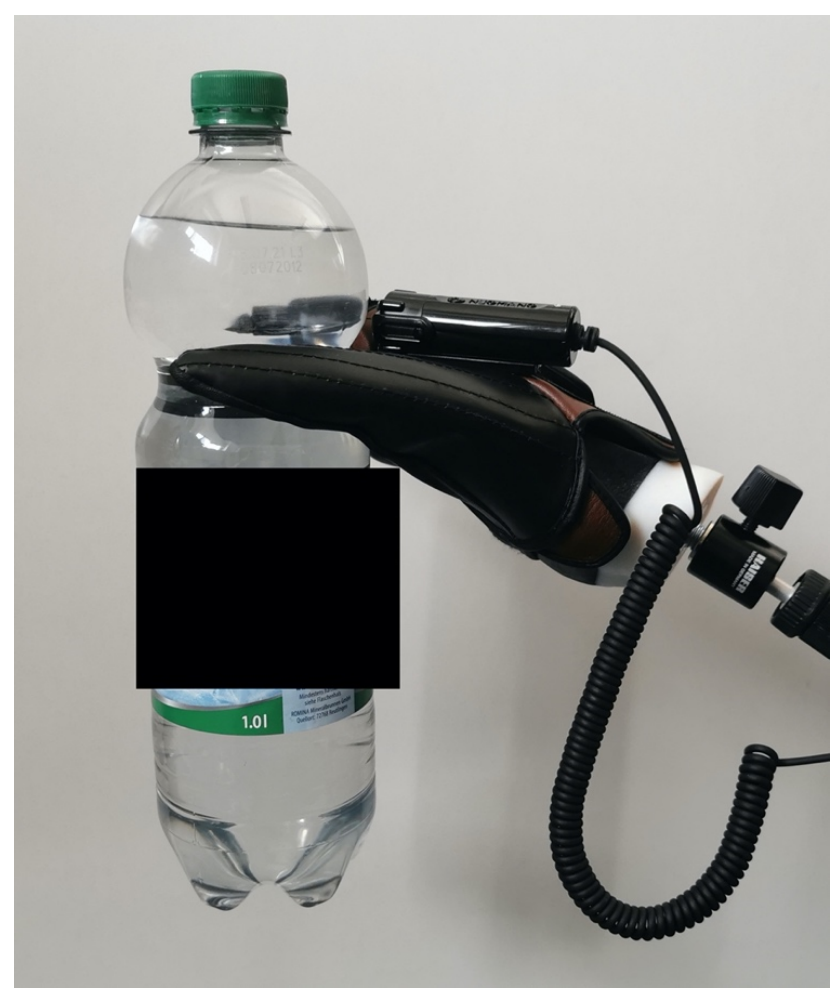

Figure 4. A full 1-L bottle is gripped and held up. 


\section{Discussion}

This work has shown that it is possible to develop a functional and cost-effective hand prosthesis with a 3D-printed replacement hand combined with a commercially available motorized orthosis. It is fascinating how many different objects can be gripped and held. In addition, 3D scanning of a volunteer's hand made it possible to meet the optical requirements of an anthropomorphic and personalized replacement hand. The resemblance of the replacement hand to the real hand is very strong. It has five fingers and, regardless of the colors of the material, it looks like a real hand due to its natural shape and features such as fingernails and tendons. Also, the weight of the replacement hand is approximately the same as the weight of the real hand.

A further step towards a natural and aesthetic prosthesis will be the adaptation of the color of the replacement hand to the natural color of the skin of the hand amputee. Aesthetics plays an important role in the successful integration of the prosthesis into the patient's everyday life [10]. However, color matching may require intensive manual labor, which could increase the cost of the hand prosthesis. Furthermore, a wide variety of particles such as dust adhere to the surface of the Agilus30 material. A possible coating of the replacement hand could prevent this. A biocompatible coating of silicone is conceivable in order to be able to use the replacement hand completely harmlessly for medical use. Another possibility would be to print the replacement hand directly with a silicone that is biocompatible.

Up to now, a complete closure of the fingers by the chosen orthosis could not be achieved. This is due to the positioning of the thumb. When closing the fingers, the fingers are pulled towards the thumb by a stable cord. If the thumb is too far away from the index finger, the index finger and middle finger are bent sideways and do not close completely. For this reason, the correct position of the fingers should be ensured during the 3D scan. Generally, the scanning process is a challenge, since the hand must ideally be held in the resting position for up to two minutes. Even small movements of the fingers can lead to faulty images. This is why a 3D scan plan should be developed to standardize the scanning process.

Currently, a remote control is needed to control the prosthesis. This requires that the user of the prosthesis have a healthy hand with which he can operate the two buttons on the remote control. A novel approach to electrodeless visual control of the orthosis with augmented reality glasses can counteract this $[11,12]$, which is worth testing.

The impressive results of this proof-of-technical-concept study encourage further clinical studies with users.

\section{Materials and Methods}

The 3D scan of a volunteer's hand (S.H.) was performed with a structured light 3D scanner (Artec Eva, Artec3D, Luxembourg). The resulting 3D model was exported as a mesh file in STL format. The resulting mesh body was then converted into a solid body using a CAD program (SolidWorks 2019, Dassault Systèmes, Vélizy-Villacoublay, France). SolidWorks was also used to separate the solid body in the area of the wrist into two parts, allowing different materials to be assigned to the two parts. A fastening system was integrated into the wrist part (Figure 5) of each replacement hand. Additionally, the skeleton was inserted into the flexible part of the second replacement hand.

The two replacement hands were printed using a multi-material 3D printer (Stratasys J750, Eden Prairie, MN, USA), which allows to print an object with several materials in one step. This approach allows to manufacture the model without additional work steps, such as the construction of casting molds. In addition, the registration of the skeleton structure in the hand can be accurately handled within the CAD program. The 3D printer utilizes the polyjet printing technique, which uses a liquid photopolymer as base material.

To select the material of the part of the hand above the wrist, individual fingers were printed with different blends of the photopolymers Agilus30 and VeroWhite. Agilus30 has similar properties to rubber in terms of appearance, haptics and function. It has a Shore-A-value of 30, making the material 
very flexible. In addition, compared to other rubber-like photopolymers, it has a higher tensile strength, a higher tear resistance and a higher elongation at break [13]. These properties are significant because the flexible part of the replacement hand must be able to withstand multiple bending and flexing processes without tearing. The photopolymer VeroWhite becomes rigid after curing [14]. By mixing the two materials with different mixing ratios, new materials with new properties are created. The more VeroWhite is added to Agilus30, the higher the Shore-A-value. As a result, the material becomes stronger, but also increasingly brittle. The printed fingers were bent manually [15]. It was observed how much force was required to achieve a full flexion of the fingers and whether tears were formed in the material during flexion. A secondary criterion was the strength of the material, although this was neglected in retrospect, since the complete flexion of the fingers was more crucial. In the end, after several manual bending processes of the fingers, the pure Agilus30 was chosen for the flexible part of the hand. The wrist part was printed with pure VeroWhite.

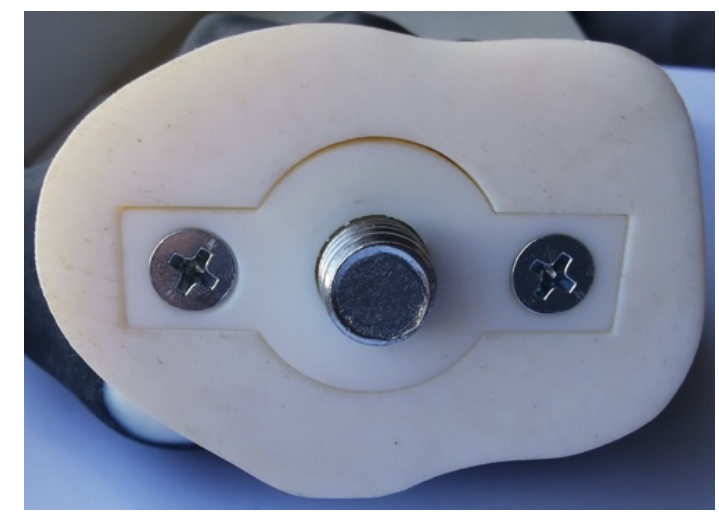

(a)

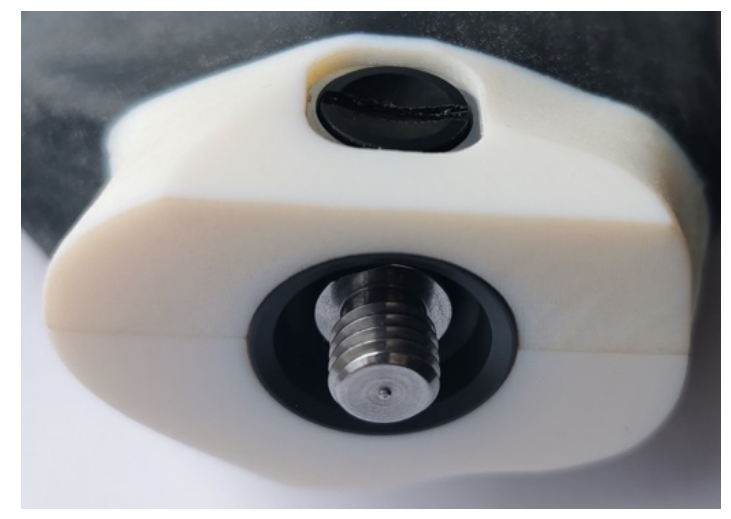

(b)

Figure 5. Replacement hands with the assembled fastening systems. (a) Fastening system with a hexagon head screw; (b) fastening system with a locking system.

The skeleton was also printed with VeroWhite. Originally, the skeleton was supposed to consist of mechanical joints and phalanges. In order to ensure that the joints can move, the areas around the joints had to be free of material. With the polyjet printing process, no overhangs can be built, so free areas are filled with a gel-like supporting material. Since the support material is inside the hand and completely surrounded by model material, it cannot be removed. Therefore, it should be used as a cartilage replacement. Nevertheless, the flexible outer material around the joints is too thin, which is why it is torn the first time the finger is bent with the orthosis. For this reason, the variant of a skeleton without joints was designed and used, in which no tears occurred despite repeated bending.

In addition, a fastening system was developed and designed for each replacement hand on the wrist, so that the replacement hands can each be mounted on a commercially available arm liner. The main part of the fastening system of the replacement hand without the integrated skeleton is an M10 threaded screw with a hexagon head, which fits into the M10 threaded hole in the arm liner. Therefore, the head of the screw must be installed in the wrist of the replacement hand. For this purpose, a counterpart has been constructed which fits exactly on the head of the screw and is screwed to the wrist. Figure 5a shows the assembled fastening system. For the fastening system of the replacement hand with the integrated skeleton, a locking system (5W055 Shuttle-Lock, Wagner Polymertechnik $\mathrm{GmbH}$, Silkerode, Germany) was used. It consists of a plastic housing with two notches, a release button and a locking pin, which in turn consists of an insert thread and an M10 threaded screw. By pressing the release button, the locking system allows the replacement hand to be attached to the liner and to be removed again. The housing of the locking system was installed in the wrist, so that the release button can be pressed from the dorsal view. The fastening system is shown in 
Figure $5 \mathrm{~b}$. Some more detailed photographs of the 3D-printed wrist of the replacement hand including the fastening systems are shown in Appendix A.

Supplementary Materials: The following are available online at http://www.mdpi.com/2673-1592/2/4/34/s1, Video S1: Functionality tests of the replacement hand in everyday situations.

Author Contributions: Conceptualization, all; methodology, all; validation, all; formal analysis, J.B.; investigation, J.B.; writing —original draft preparation, J.B.; writing-review and editing, S.H. and A.O.; visualization, J.B.; supervision, S.H. and A.O.; project administration, A.O. All authors have read and agreed to the published version of the manuscript.

Funding: This research received no external funding.

Conflicts of Interest: The authors declare no conflict of interest. This paper contains parts of the Bachelor's thesis of J.B., which was supervised by S.H. and A.O., 2020 (see [15]).

\section{Appendix A}

In Appendix A, some more detailed photographs of the 3D-printed wrist of the replacement hand including the fastening systems are shown (Figures A1 and A2).

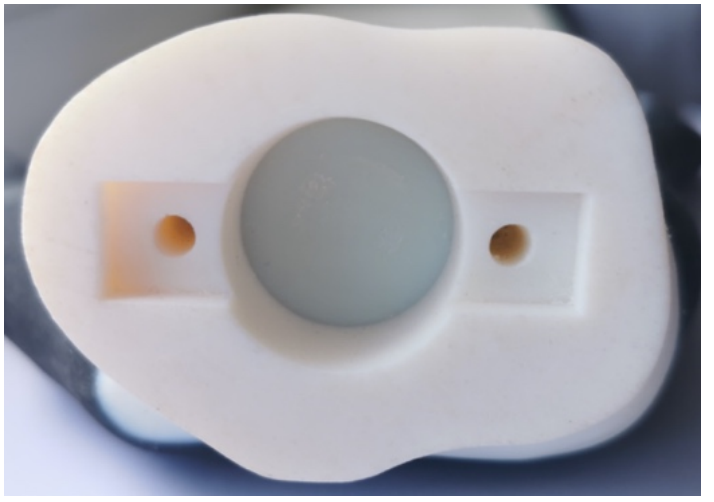

(a)

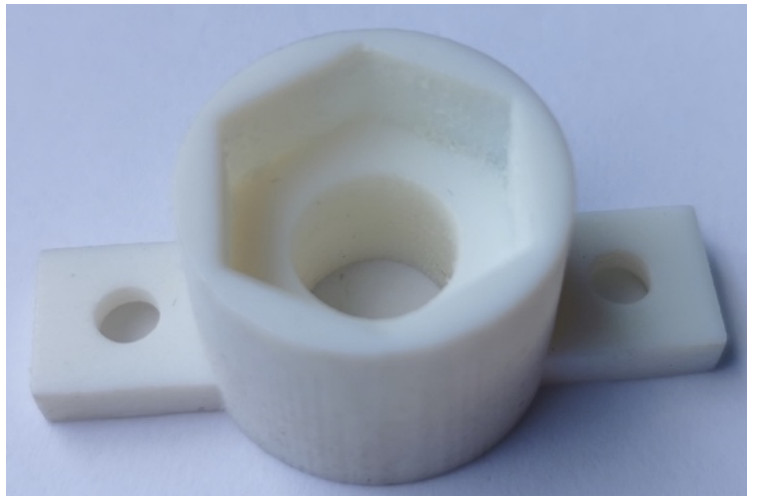

(b)

Figure A1. Fastening system with a hexagon head screw. (a) Counterpart for the hexagon head screw; (b) wrist of the replacement hand with a recess for the hexagon head screw and the counterpart.

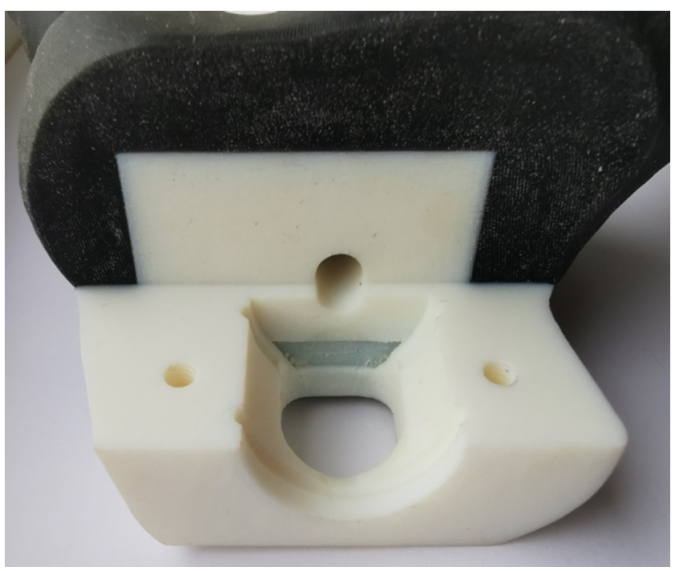

(a)

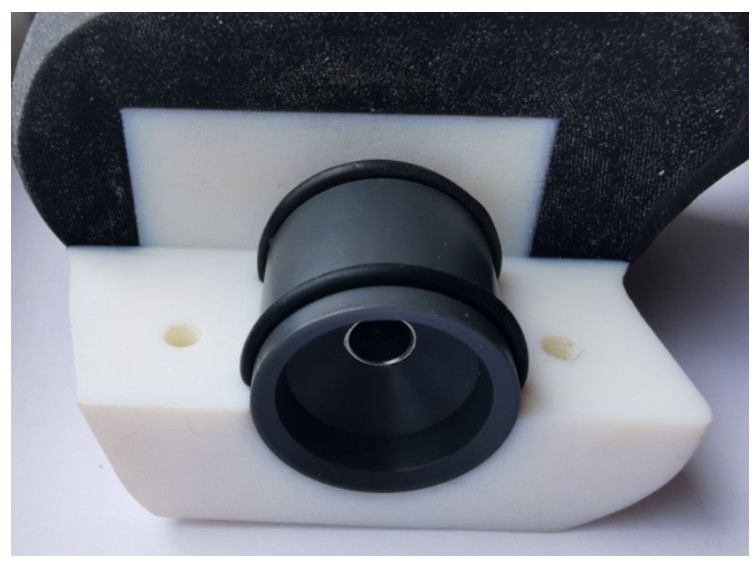

(b)

Figure A2. Cont. 


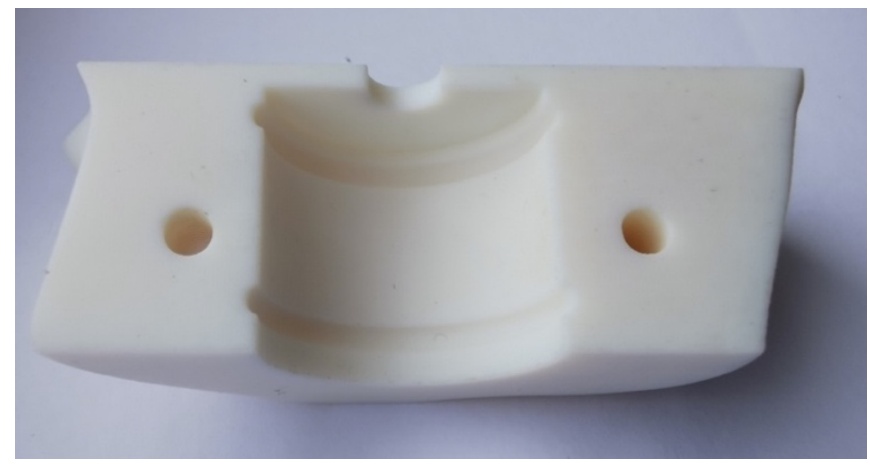

(c)

Figure A2. Fastening system with a locking system. (a) First part of the wrist with a recess for the locking system; (b) the locking system is installed in the wrist; (c) second part of the wrist.

\section{References}

1. Bouton, C.E.; Shaikhouni, A.; Annetta, N.V.; Bockbrader, M.A.; Friedenberg, D.A.; Nielson, D.M.; Sharma, G.; Sederberg, P.B.; Glenn, B.C.; Mysiw, W.J.; et al. Restoring cortical control of functional movement in a human with quadriplegia. Nature 2016, 533, 247-250. [CrossRef] [PubMed]

2. Soekadar, S.R.; Witkowski, M.; Gómez, C.; Opisso, E.; Medina, J.; Cortese, M.; Cempini, M.; Carrozza, M.C.; Cohen, L.G.; Birbaumer, N.; et al. Hybrid EEG/EOG-based brain/neural hand exoskeleton restores fully independent daily living activities after quadriplegia. Sci. Robot. 2016, 1. [CrossRef] [PubMed]

3. McMullen, D.P.; Hotson, G.; Katyal, K.D.; Wester, B.A.; Fifer, M.S.; McGee, T.G.; Harris, A.; Johannes, M.S.; Vogelstein, R.J.; Ravitz, A.D.; et al. Demonstration of a semi-autonomous hybrid brain-machine interface using human intracranial EEG, eye tracking, and computer vision to control a robotic upper limb prosthetic. IEEE Trans. Neural Syst. Rehabil. Eng. 2014, 22, 784-796. [CrossRef]

4. Otte, A. Smart Neuroprosthetics Becoming Smarter, but Not for Everyone? EClinicalMedicine 2018, 2-3, 11-12. [CrossRef]

5. Egermann, M.; Kasten, P.; Thomsen, M. Myoelectric hand prostheses in very young children. Int. Orthop. 2009, 33, 1101-1105. [CrossRef]

6. Otte, A. Invasive versus Non-Invasive Neuroprosthetics of the Upper Limb: Which Way to Go? Prosthesis 2020, 2, 237-239. [CrossRef]

7. Chadwell, A.; Kenney, L.; Howard, D.; Ssekitoleko, R.T.; Nakandi, B.T.; Head, J. Evaluating Reachable Workspace and User Control over Prehensor Aperture for a Body-Powered Prosthesis. IEEE Trans. Neural Syst. Rehabil. Eng. 2020, 28, 2005-2014. [CrossRef] [PubMed]

8. Koprnicky, J.; Najman, P.; Safka, J. 3D printed bionic prosthetic hands. In Proceedings of the IEEE International Workshop of Electronics, Control, Measurement, Signals and Their Application to Mechatronics, ECMSM, Donostia, San Sebastian, Spain, 24-26 May 2017. [CrossRef]

9. Otte, A. 3D Computer-Aided Design Reconstructions and 3D Multi-Material Polymer Replica Printings of the First "Iron Hand" of Franconian Knight Gottfried (Götz) von Berlichingen (1480-1562): An Overview. Prosthesis 2020, 2, 304-312. [CrossRef]

10. Leow, M.E.L.; Ow, R.K.K.; Lee, M.H.; Huak, C.Y.; Pho, R.W.H. Assessment of colour differences in silicone hand and digit prostheses: Perceptible and acceptable thresholds for fair and dark skin shades. Prosthet. Orthot. Int. 2006, 30, 5-16. [CrossRef] [PubMed]

11. Hazubski, S.; Hoppe, H.; Otte, A. Non-contact visual control of personalized hand prostheses/exoskeletons by tracking using augmented reality glasses. 3D Print. Med. 2020, 6, 20-22. [CrossRef] [PubMed]

12. Hazubski, S.; Hoppe, H.; Otte, A. Electrode-free visual prosthesis/exoskeleton control using augmented reality glasses in a first proof-of-technical-concept study. Sci. Rep. 2020, 10, 1-10. [CrossRef] [PubMed]

13. Stratasys. Available online: https://www.stratasys.com/de/materials/search/agilus30 (accessed on 21 November 2020).

14. Stratasys. Available online: https://www.stratasys.com/de/materials/search/vero (accessed on 21 November 2020). 
15. Baron, J. 3D-Druck Einer Anthropomorphen Ersatzhand für die Neuroprothetik [3D Printing of an Anthropomorphic Artificial Hand for Neuroprosthetics]. Bachelor's Thesis, Offenburg University, Offenburg, Germany, 31 August 2020.

Publisher's Note: MDPI stays neutral with regard to jurisdictional claims in published maps and institutional affiliations.

(C) 2020 by the authors. Licensee MDPI, Basel, Switzerland. This article is an open access article distributed under the terms and conditions of the Creative Commons Attribution (CC BY) license (http://creativecommons.org/licenses/by/4.0/). 